\title{
Cardiometabolic Risk in Patients with Schizophrenia: A Literature Review
}

Zanola, M. 1; Galati, M.S. ; Rahe, B.B. ${ }^{2}$

${ }^{1}$ Santa Marcelina College, Medical School, São Paulo, Brazil.

2Santa Marcelina College, Department of Psychiatry, São Paulo, Brazil

Objectives:

To evaluate the factors that contribute to the increase of the cardiometabolic risk and consequent mortality in patients with schizophrenia and how unhealthy lifestyle habits contribute to the metabolic risk in these patients. Also investigate which characteristics of the antipsychotics contribute to cardiometabolic risk.

\section{Background:}

According to the World Health Organization's definition, schizophrenia is a severe mental disorder, characterized by deep ruptures of thinking, with impairment in language, perception and sense of self, affecting about $1 \%$ of the world population. ${ }^{1}$ Patients with schizophrenia have disproportionate rates of morbidity and mortality compared to the rest of the population. The overall mortality rate is two or three times higher for these patients, with life expectancy of about 20 years less than the general population. ${ }^{2}$ Among the causes of early mortality is the high risk of suicide and lifestyle factors such as unhealthy diet, smoking, alcohol abuse and sedentary lifestyle. Overweight, obesity and metabolic syndrome are common in schizophrenia, particularly in association with treatment using antipsychotic drugs. These patients have, therefore, a higher risk of adverse events from cardiovascular and cerebrovascular diseases. ${ }^{3}$

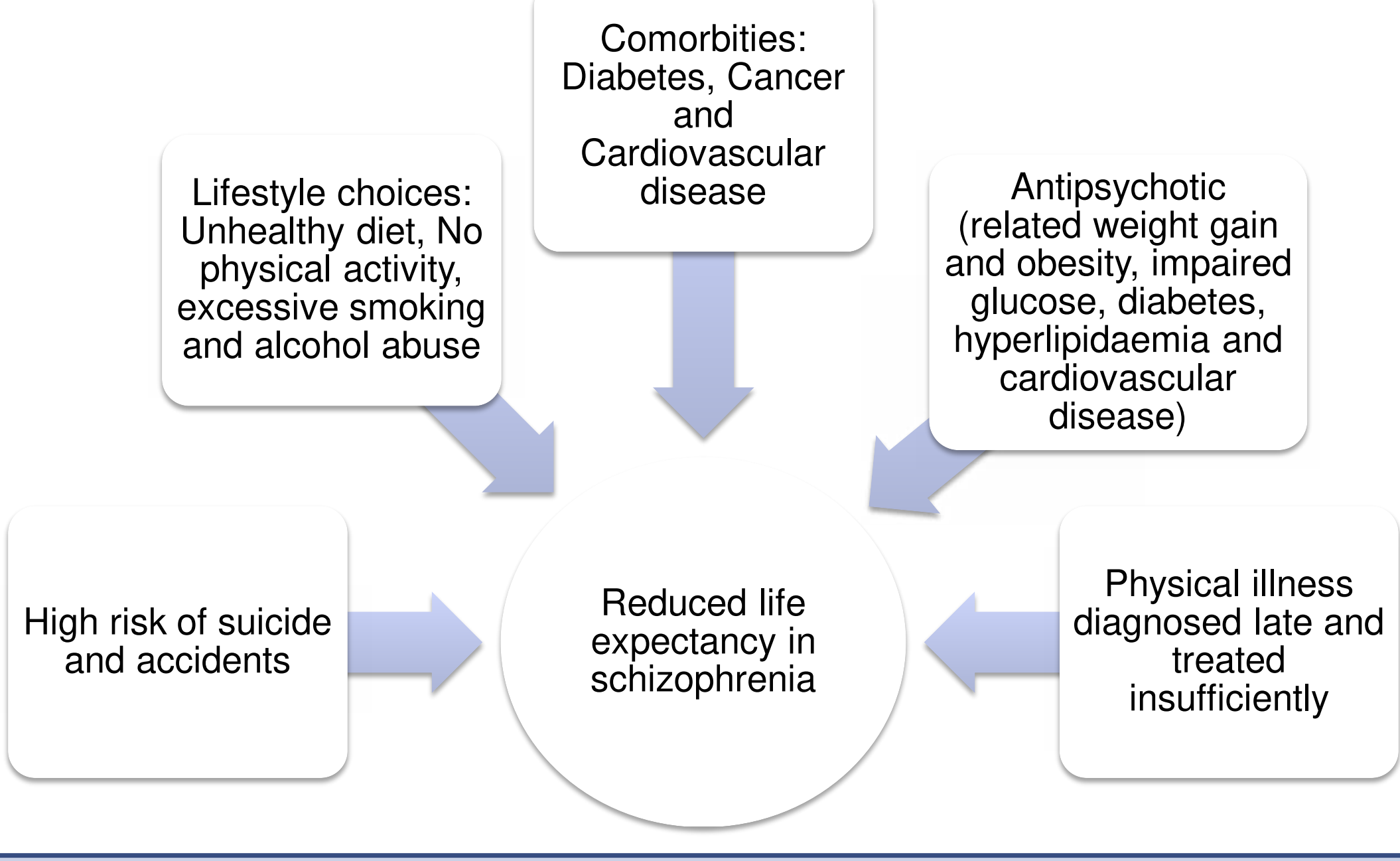

Materials and Methods:

The study is a systematic review, containing the synthesis of several published studies that allows general conclusions about the theme. The following steps were followed: problem formulation, data collection, evaluation, analysis and interpretation of collected data and presentation of results. Our research strategy included the terms cardiometabolic risk AND schizophrenia, in all fields of knowledge, to be as broad as possible. The terms were searched in articles in the following database: MEDLINE, Web of Science and Cochrane.

\section{Results and Conclusions:}

The increase in cardiovascular risk in the population with schizophrenia can be attributed to many factors, but the main one is the grouping characteristics that define the metabolic syndrome: central obesity, hypertension, altered glycemia or diagnosis of diabetes and dyslipidemias. ${ }^{4}$ Some studies identified specific inflammatory markers present in these patients, such as TNFalpha, interleukin 6, and CRP, and they all play a part in the development of insulin resistance, type 2 diabetes and metabolic syndrome. ${ }^{5}$ The adverse effects of antipsychotic treatment have been reported as contributing to the increase in cardiometabolic risk, affecting lipid and glucose metabolisms with some known and unknown mechanisms. The first- and second-generation antipsychotics can cause significant weight gain, diabetes mellitus and cardiovascular diseases. Evidence shows that treatment with these medications may increase adiposity, the risk of hyperglycemia and alter plasma lipids. ${ }^{6}$ Even though genetic factors might have a role in the physical health problems of patients with schizophrenia, environmental and lifestyle factors such as little physical activity, smoking and unhealthy diet play an important part. These individuals have a poor diet that partly accounts for their higher incidence of metabolic abnormalities and along with harmful lifestyle choices, such as cigarette smoking and no exercise, are factors strongly associated and a major contributor to cardiovascular diseases. ${ }^{5}$ Weight gain is a sign that is commonly used as a marker of cardiometabolic risk associated with schizophrenia and its treatment. This is because weight gain is an early marker because the changes are evident in the weeks of initiation of treatment. It's also an obvious change for patients, their families and their doctors, being easily measured in the clinic. ${ }^{7}$ Physical illnesses in people with schizophrenia, such as cardiovascular and metabolic diseases, are common, but diagnosed late and treated insufficiently. ${ }^{8}$ It's convenient to study these patients with emphasizing in primary care, in order to be able to define exactly the strategies to be followed, and thus to obtain the greatest benefit, without forgetting the fragility that surrounds this group. ${ }^{9}$ Therefore, care coordinators may help to bridge the gap between physical and mental health, and the primary care physicians may be central in defining the needs and coordinating the different initiatives. ${ }^{8}$ All variables that moderate cardiometabolic risk in healthy individuals have the potential to moderate it in patients with schizophrenia. For this reason, it is important that both internist and the psychiatrist be aware of the risks these patients may have for proper planning of the appropriate lifestyle and pharmacological interventions.

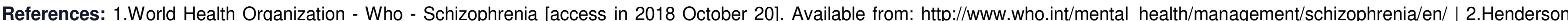

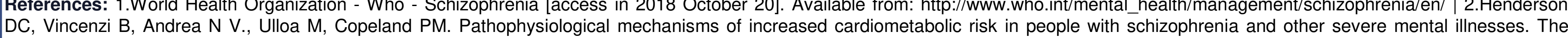

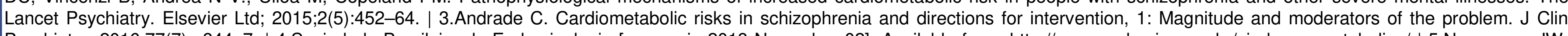

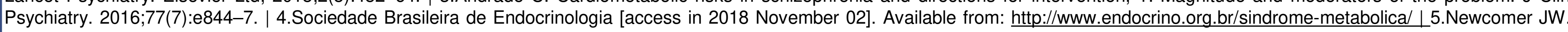

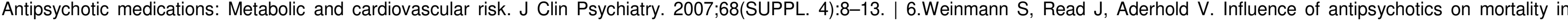

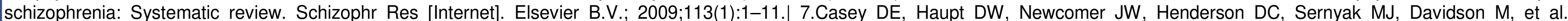

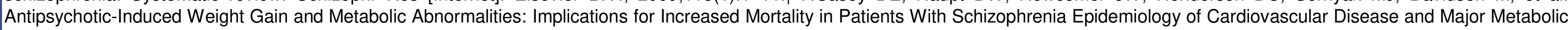

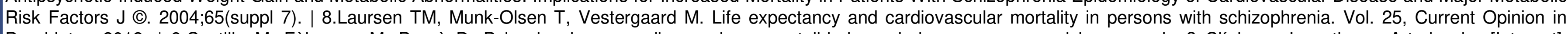

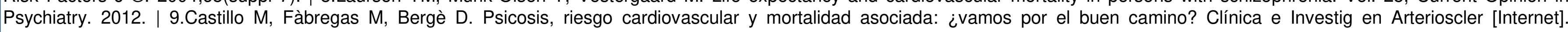
2013;(January 2018). 